\title{
Potential energy curves and dipole transition moments for excited electronic states of $\mathrm{XeKr}$ and ArNe
}

\author{
loannis D. Petsalakis and Giannoula Theodorakopoulos ${ }^{\text {a) }}$ \\ Theoretical and Physical Chemistry Institute, The National Hellenic Research Foundation, \\ 48 Vassileos Constantinou Ave., Athens 11635 Greece \\ Heinz-Peter Liebermann and Robert J. Buenker \\ University of Wuppertal, Fachbereich 9, Theoretische Chemie, Gaussstr. 20, D-42097 Wuppertal, Germany
}

(Received 6 March 2002; accepted 29 May 2002)

\begin{abstract}
Relativistic core-potential calculations have been carried out on $\Omega$ states resulting from the interaction of $\mathrm{Xe}^{*}\left(5 p^{5} 6 s,{ }^{3} P,{ }^{1} \mathrm{P}\right)$ with ground-state $\mathrm{Kr}$ atoms as well as for the system $\mathrm{Ar}^{*}$ $\left(3 p^{5} 4 s,{ }^{3} P,{ }^{1} P\right)$ with ground-state $\mathrm{Ne}$, using different basis sets and configuration interaction procedures. The present calculations on ArNe, employing larger sets of Rydberg functions than those of the previous calculations, yield totally repulsive potentials for the excited states of ArNe. Similar calculations on XeKr obtain shallow minima $\left(600-860 \mathrm{~cm}^{-1}\right)$ in the potential energy curves of the excited states at large internuclear distances (6.9-7.8 bohr). Dipole transition moments have been calculated and strong radiative transitions are predicted from excited states to the ground state. The 1(I) state, correlating with the metastable ${ }^{3} P_{2}$ state of $\mathrm{Xe}$ is found to have a small but nonzero dipole transition moment at short and intermediate nuclear distances leading to a radiative lifetime for the $v=0$ level of this state of $21.0 \mu \mathrm{s}$. (C) 2002 American Institute of Physics.
\end{abstract}

[DOI: 10.1063/1.1494796]

\section{INTRODUCTION}

The excited states of diatomic rare-gas molecules are important for their role as radiation sources in the vacuum ultraviolet (VUV) region, including excimer lasers. ${ }^{1-3}$ While most experimental and theoretical work has been devoted to homonuclear systems, the heteronuclear systems have also attracted interest following the observation of very efficient energy transfer processes in admixtures of rare gases, ${ }^{1,4-6}$ and this opens new possibilities for the efficient pumping of these systems. ${ }^{7}$ The advent of experimental studies of thermal energy collisions between excited and ground-state raregas atoms ${ }^{8,9}$ offers a challenge to theoreticians to provide interpretations of the resulting experimental data. Accurate potential energy curves are required for the interacting pairs of atoms over a large range of interatomic distances. However, despite the conceptual simplicity of their electronic structure, accurate determinations of the interaction potentials for the heteronuclear dimers are scarce due to computational difficulties involved. The precise determination of the shallow minima generally found in these systems require large configuration interaction (CI) expansions and adequate basis sets, especially in the Rydberg part. Often, minima which have been proposed in experimental work are not found in the calculations, as for example in the excited states of $\mathrm{Kr}-\mathrm{Ar},{ }^{10}$ while errors of $\pm 300 \mathrm{~cm}^{-1}$ in the calculated potential wells are not unexpected. ${ }^{11}$ Furthermore, it is essential to include spin-orbit coupling in order to calculate the electronic states correctly. The ground-state potential has different requirements from the excited states in terms of polar-

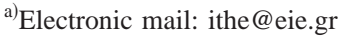

ization functions and exponents of the diffuse functions. Good potentials for the ground states of the $\mathrm{RgRg}^{\prime}$ systems have been reported and one could focus on the excited-state requirements. ${ }^{12,13}$

Appropriate $a b$ initio methods to calculate potential energy curves for the electronic states of these molecules make use of relativistic core potentials and such calculations have been reported for heteronuclear systems such as $\mathrm{KrAr},{ }^{10}$ ArNe, ${ }^{14} \mathrm{XeHe}$ and $\mathrm{XeAr}^{11}$ and more recently ArHe and HeNe. ${ }^{15}$ The calculations on ArHe showed that for such systems it is necessary to employ at least a double-zeta Rydberg basis on the heavier atom in order to calculate the potential energy curves free from spurious "bumps" and minima. The previous work on ArNe by the present authors ${ }^{14}$ employed a single Rydberg $s$ and a single Rydberg $p$ function on Ar with the same exponent, which had been optimized with respect to the atomic Ar excitation energy. While this approach resulted in good agreement at the dissociation limits for the atomic excitation energies and also for transition moments, it was found to lead to erroneous results at short and intermediate internuclear distances for the $\mathrm{Ar}^{*}+\mathrm{He}$ system. ${ }^{15}$ For this reason, it became necessary to re-calculate the potentials for the $\mathrm{Ar}^{*}\left(3 p^{5} 4 s\right)+\mathrm{Ne}$ interaction in the present work, and to correct the previously reported potentials.

The $\mathrm{Xe}^{*}\left(5 p^{5} 6 s\right)+\mathrm{Kr}$ system, which is the main object of the present work, has been studied by spectroscopic methods, ${ }^{1-3,7,16-22}$ while potential energy curves have been calculated by model Hamiltonian methods. ${ }^{23,24}$ Work based on absorption spectra of Xe-rare gas mixtures showed the existence of a quasi-bound XeKr excimer, near $1469.61 \AA$, with a well depth of 120 or $166 \mathrm{~cm}^{-1}$ at an internuclear distance of 4.78 or $4.36 \AA$, depending on the potential pa- 
rameters adopted ${ }^{5}$ and elsewhere, another estimate ${ }^{3}$ of the well depths as $153 \mathrm{~cm}^{-1}$ for the excited state near the first resonanance line of Xe I and $333 \mathrm{~cm}^{-1}$ for the state near the second resonanance line of Xe I (at $1295.59 \AA)^{3}$. Analysis of laser induced fluorescence (LIF) spectra involving transitions from the ground state to the states $0^{+}\left({ }^{3} P_{1}\right)$ and $1\left({ }^{3} P_{1}\right)$ of $\mathrm{Xe} * \mathrm{Kr}$ indicated a double well potential for the $0^{+}$state (with depths of 624 and $101 \mathrm{~cm}^{-1}$ at $r_{e}$ of 3.09 and $5.1 \AA$ ) and a very shallow well for the 1 state $\left(52 \mathrm{~cm}^{-1}\right.$ at $\left.5.24 \AA\right) .{ }^{19}$ Emission spectroscopy work of solid $\mathrm{Kr}$ bombarded with ${ }^{3} P_{2}$ Xe atoms ${ }^{16}$ has reported emission at $7.95 \mathrm{eV}$ corresponding to the $\mathrm{XeKr} 1\left({ }^{3} P_{2}\right)$ exciplex with a binding energy of $138 \pm 50 \mathrm{~cm}^{-1}$ at $r_{e}=3.20 \pm 0.05 \AA$. Luminescence studies of the $\mathrm{XeKr}$ exciplex in liquid and in solid krypton obtained a lifetime of $52 \pm 3 \mathrm{~ns}$ in the liquid and $50 \pm 3 \mathrm{~ns}$ in the solid which were assigned to the $1\left({ }^{3} P_{2}\right)$ exciplex. ${ }^{17}$ However, it is more likely that the reported data should be assigned to the $0^{+}\left({ }^{3} P_{1}\right)$ exciplex. ${ }^{25}$ Model potential calculations predict deeper minima for the exciplex states, ranging from $1000-1200 \mathrm{~cm}^{-1}$ [for the $0^{-}\left({ }^{3} P_{2}\right), 1\left({ }^{3} P_{2}\right)$, and $0^{+}\left({ }^{3} P_{1}\right)$ states] and around $300 \mathrm{~cm}^{-1}$ [for the $2\left({ }^{3} P_{2}\right)$ and $1\left({ }^{3} P_{1}\right)$ states $],{ }^{23-25}$ while simulations of absorption spectra employing Morse potentials with parameters obtained from the above calculations found $170 \mathrm{~cm}^{-1}$ to be a more suitable value for the well-depth of the $1\left({ }^{3} P_{1}\right)$ state. ${ }^{22}$ Thus it is a rather confusing situation, considering that the emission spectra of $\mathrm{XeKr}$ overlap with those of the homonuclear dimer, $\mathrm{Xe}_{2}$ and the fact that the excited states corresponding to a particular limit are closely lying and also lead to overlapping emission spectra. ${ }^{1,21,22}$ Similarly for the next higher states, correlating with excited $\mathrm{Xe}+$ ground-state $\mathrm{Kr}$ limits, besides the estimated well depth of $333 \mathrm{~cm}^{-1}$ mentioned above, ${ }^{3}$ Tsuchizawa et al. ${ }^{18}$ have reported minima of 1445 and $54 \mathrm{~cm}^{-1}$ for the $1\left({ }^{1} P_{1}\right)$ and the $0^{+}\left({ }^{1} P_{1}\right)$ states, respectively, while Mao et al. ${ }^{20}$ propose the opposite assignments. Thus despite the aforementioned computational problems, a very careful theoretical study on these systems could shed some light on these issues.

In the present work, multireference configuration interaction calculations (MRDCI) employing relativistic effective core potentials (RECP) have been carried out on $\Omega$ states resulting from the interaction of $\mathrm{Xe}^{*}\left(5 p^{5} 6 s,{ }^{3} P,{ }^{1} P\right)$ with ground-state $\mathrm{Kr}$ atoms as well as for the system $\mathrm{Ar}^{*}$ $\left(3 p^{5} 4 s,{ }^{3} P,{ }^{1} P\right)$ with ground-state $\mathrm{Ne}$, using a variety of basis sets and configuration interaction procedures. Potential energy curves and dipole transition moments have been calculated and radiative lifetimes of the excited states have been determined.

\section{CALCULATIONS}

The present calculations on $\mathrm{XeKr}$ include the ground and excited electronic states correlating with the limits $\mathrm{Xe}$ $\left(5 p^{5} 6 s,{ }^{3} \mathrm{P},{ }^{1} \mathrm{P}\right)$ plus ground-state $\mathrm{Kr}$, and similarily those on ArNe include the ground and excited states correlating with $\operatorname{Ar}\left(3 p^{5} 4 s,{ }^{3} P,{ }^{1} P\right)$ plus ground-state Ne. In $C_{2 V}$ symmetry, the resulting states in each case comprise two ${ }^{1} A_{1}$ states and one of ${ }^{3} A_{1},{ }^{1} B_{1}$ and ${ }^{1} B_{2},{ }^{3} B_{1}$, and ${ }^{3} B_{2}$ symmetry, which correspond to the lowest two ${ }^{1} \Sigma^{+}$, and the lowest ${ }^{3} \Sigma^{+},{ }^{1} \Pi$, and ${ }^{3} \Pi$ states, respectively. These states give rise to $\Omega$ states of $0^{+}(3), 0^{-}(2), 2(1), 1(3)$ symmetry in the full linear double group and of total $C_{2 V}$ symmetry $A_{1}, A_{2}, B_{1}$, and $B_{2} \cdot{ }^{10,14,15}$

The calculations have been carried out with the aid of a relativistic effective core potentials (RECP) version of the MRDCI programs, using the contracted CI implementation $^{26-30}$ which involves a two-step procedure. In the first step $\Lambda$-S electronic states are determined in conventional CI calculations in which all the electronic integrals are calculated with the aid of RECPs, whereby the self-consistent field-molecular orbital (SCF-MO) basis is computed in a treatment which includes only the scalar relativistic terms in addition to the conventional nonrelativistic Hamiltonian. The resulting $\Lambda$-S states are employed in the second step to form the full Hamiltonian matrix including the spin-orbit interaction. Diagonalization is then carried out for each total symmetry to determine eigenvalues and eigenfunctions. In the present work, the latter are in turn employed for the computation of dipole transition moments between electronic states. These calculations are carried out for different values of the internuclear distance $R$, varying from 4.0 to $20.0 \mathrm{bohr}$ for $\mathrm{XeKr}$ and from 3.5 to $20.0 \mathrm{bohr}$ for ArNe and also for $R$ $=100 \mathrm{bohr}$.

Relativistic core potentials are employed for both $\mathrm{Xe}(\mathrm{K}$, $\mathrm{L}, \mathrm{M}$, and $\mathrm{N}$ shells $)^{31}$ and $\mathrm{Kr}$ (K, L, and M shells). ${ }^{32}$ For each atom the Gaussian basis sets for the valence shell ${ }^{31,32}$ were augmented with two sets of $d$ and one set of $\mathrm{f}$ polarization functions with exponents $0.31,0.15$, and 0.5 , respectively, for Xe and 0.5, 0.3, and 0.12, respectively, for Kr. The necessary diffuse functions were also included, three $s$ (exponents $0.09,0.055,0.021$ ), three $p$ (exponents 0.07, 0.036, 0.013 ), and one $d$ (exponent 0.058 ) in the Xe basis set and two s (exponents $0.1,0.05$ ) and two $p$ (exponents 0.07, 0.04) in the $\mathrm{Kr}$ basis set. Several sets of calculations were carried out employing different diffuse and/or polarization functions and $\mathrm{CI}$ procedures in order to establish convergence of the calculated potentials, at least in the qualitative features. The above basis set, with the valence Gaussian functions included in uncontracted form, has been employed to obtain the results that will be discussed in the present work.

In multireference calculations, the choice of reference space is very important because it determines the zerothorder description of the states, and the CI spaces are generated by allowing single and double excitations with respect to all the reference configurations. The reference spaces employed in the present work, consisting of nine configurations for the ${ }^{1} A_{1}$ and eight configurations for each of the ${ }^{3} A_{1}$, ${ }^{1} B_{1},{ }^{3} B_{1},{ }^{1} B_{2}$ and ${ }^{3} B_{2}$ calculations, have been determined by test calculations at different values of the internuclear distance in each case and they characterize the calculated wave functions throughout with a contribution of over $90 \%$. Exploratory calculations in the present work indicate that it is desirable to have $T=0$ calculations for the potentials, as was also found in our previous work on ArHe and HeNe. ${ }^{15}$ For this reason and in order to keep the calculations tractable, the highest energy $14 a_{1}, 8 b_{1}, 8 b_{2}$ and $3 a_{2}$ virtual orbitals were not included in the CI. This left $22 a_{1}, 12 b_{1}$, $12 b_{2}$ and $4 a_{2} \mathrm{MO}$ for the 16-electron CI calculations. The CI spaces include all the configuration functions resulting 
TABLE I. Energy levels of $\operatorname{Xe}\left(5 p^{6}{ }^{1} S, 5 p^{5} 6 s^{1,3,} P\right)+\operatorname{Kr}\left(2 p^{6}{ }^{1} S\right)$ and $\operatorname{Ar}\left(3 p^{6}{ }^{1} S, 3 p^{5} 4 s^{1,3,} P\right)+\operatorname{Ne}\left(2 p^{6}{ }^{1} S\right)$ at $100.0 \mathrm{bohr}$.

\begin{tabular}{|c|c|c|c|c|}
\hline \multirow[b]{2}{*}{$\Omega$ states } & \multicolumn{2}{|c|}{$\mathrm{XeKr}$} & \multicolumn{2}{|c|}{$\mathrm{ArNe}$} \\
\hline & $\begin{array}{c}\Delta E^{\mathrm{a}} \text { present work } \\
\mathrm{cm}^{-1}\end{array}$ & $\begin{array}{c}\Delta E \underset{\mathrm{em} \text { expimental }}{\mathrm{cm}^{-1}} \\
\text { ent }\end{array}$ & $\begin{array}{c}\Delta E^{\mathrm{b}} \text { present work } \\
\mathrm{cm}^{-1}\end{array}$ & $\underset{\mathrm{cm}^{-1}}{\Delta E \text { experimental }^{\mathrm{c}}}$ \\
\hline $0^{+}(\mathrm{I})$ & 0.0 & $0.0(J=0)$ & 0.0 & $0.0(J=0)$ \\
\hline $0^{-}(\mathrm{I})$ & 67065 & & 93138 & \\
\hline $1(\mathrm{I})$ & 67065 & & 93139 & \\
\hline $2(\mathrm{I})$ & 67068 & $67068(J=2)$ & 93144 & $93143.8(J=2)$ \\
\hline $0^{+}(\mathrm{II})$ & 68126 & & 93750 & \\
\hline $1(\mathrm{II})$ & 68249 & $68046(J=1)$ & 93779 & $93750.6(J=1)$ \\
\hline $0^{-}(\mathrm{II})$ & 76474 & $76197(J=0)$ & 94553 & $94553.7(J=0)$ \\
\hline $0^{+}(\mathrm{III})$ & 77119 & & 95389 & \\
\hline $1(\mathrm{III})$ & 77023 & $77186(J=1)$ & 95369 & $95399.9(J=1)$ \\
\hline
\end{tabular}

${ }^{a}$ Excited states shifted by $1731 \mathrm{~cm}^{-1}$.

${ }^{b}$ Excited states shifted by $3409 \mathrm{~cm}^{-1}$.

${ }^{\mathrm{c}}$ Reference 37.

from all single and double excitations with respect to the reference configurations. The resulting CI spaces consist of 211553 configuration functions ( $S^{2}$ eigenfunctions) for the ${ }^{1} A_{1}, 346984$ for the ${ }^{3} A_{1}, 205712$ for the ${ }^{1} B_{1}$ and the ${ }^{1} B_{2}$ and 350480 configuration functions for the ${ }^{3} B_{1}$ and the ${ }^{3} B_{2}$ calculations. A full-CI correction ${ }^{33}$ was applied to the eigenvalues.

The present calculations on ArNe also employed relativistic core potentials for $\mathrm{Ar}(\mathrm{K}$ and $\mathrm{L}$ shells) and $\mathrm{Ne}(\mathrm{K}$ shell). ${ }^{34}$ The atomic orbital (AO) basis set employed for $\mathrm{Ar}$ is the $(12 s 9 p / 6 s 5 p)$ basis of McLean and Chandler, ${ }^{35}$ augmented with one set of $d$ functions for polarization (exponent 0.736 ) and three $s$ and one $p$ diffuse functions with exponents $0.08,0.04$ and 0.015 and 0.0405 , respectively. It was found necessary to employ such a triple-zeta basis for the $4 \mathrm{~s}$ function of $\mathrm{Ar}$ in order to obtain convergence in the resulting potentials. For Ne the $(11 s 6 p / 5 s 4 p)$ basis of Dunning ${ }^{36}$ was augmented with one polarization $d$ (exponent 0.8 ) and one $s$ and one $p$ diffuse (exponents 0.03 and 0.025 , respectively) functions. As in the above calculations, the CI strategy was to carry out $T=0$ calculations, to insure as uniform a description over the different internuclear distances as possible. To achieve this, the highest $8 a_{1}, 3 b_{1}$, and $3 b_{2}$ virtual MO were removed. Again 16-electron CI calculations were carried out employing reference spaces of eight configurations for the ${ }^{1} A_{1}$ and seven configurations for each of the ${ }^{3} A_{1}$, ${ }^{1} B_{1},{ }^{3} B_{1},{ }^{1} B_{2}$, and ${ }^{3} B_{2}$ calculations. The CI spaces included 177880 configuration functions for the ${ }^{1} A_{1}$ calculation, 174110 for the ${ }^{1} B_{1}$ and ${ }^{1} B_{2}, 288896$ for the ${ }^{3} A_{1}$ and 296233 configuration functions for the ${ }^{3} B_{1}$ and ${ }^{3} B_{2}$ calculations.

The computed excitation energies at the internuclear distance of $100.0 \mathrm{bohr}$ for both $\mathrm{XeKr}$ and ArNe are listed in Table I, along with the experimental values ${ }^{37}$ for the levels of $\mathrm{Ar}^{*}$ and $\mathrm{Ne}^{*}$, respectively. The theoretical levels have been shifted upward by $1731 \mathrm{~cm}^{-1}$ for XeKr and by $3409 \mathrm{~cm}^{-1}$ for $\mathrm{ArNe}$, as the calculations tend to favor the Rydberg states over the ground state, for which there is more correlation energy to be accounted for. This is a typical practice in such calculations. ${ }^{10,15}$ As shown in Table I, the calculated splittings of the $\mathrm{Xe}^{*}\left(5 p^{5} 6 s,{ }^{3} P,{ }^{1} P\right)$ and the
$\operatorname{Ar*}\left(3 p^{5} 4 s,{ }^{3} P,{ }^{1} P\right)$ levels are in excellent agreement with the corresponding experimental values.

\section{RESULTS AND DISCUSSION}

\section{A. Results of calculations on $\mathrm{XeKr}$}

In Table II the energies of the $\Omega$ excited states of $\mathrm{XeKr}$ calculated in the present work are given in $\mathrm{cm}^{-1}$ and with respect to the minimum energy of the ground state. These energies are as obtained after the final diagonalization including the spin-orbit coupling and do not include the shift by $1731 \mathrm{~cm}^{-1}$ mentioned above. The $\Lambda$-S potentials are given in Fig. 1 while the corresponding $\Omega$ potentials are given in Fig. 2 and finer details for some of these states are given in Figs. 3 and 4. As shown in Fig. 1, the $1^{3} \Sigma^{+}$and the $2^{1} \Sigma^{+} 5 p \sigma \rightarrow 6 s$ states have Rydberg minima for $R$ $=5.8 \mathrm{bohr}$, close to the minimum of the ground state of the cation $\mathrm{XeKr}^{+},{ }^{38}$ while the $1^{3} \Pi$ and the $1^{1} \Pi$ states show rather shallow and wide minima at larger $R$, close to 8.0 bohr. The corresponding $\Omega$ potentials, shown in Fig. 2 along with the $\Lambda$-S potentials for comparison, have notably shallower minima occurring at larger $R$ than the potentials of the $\Lambda$-S states. As shown in Fig. 2, the spin-orbit coupling has a profound effect on the relative positions of the electronic states. In Fig. 3, enlarged plots of the $0^{-}$(I), 1(I), 2(I), 1(II), and $0^{+}$(II) potentials in the region around the energy minima are given. The present results are in qualitative agreement with the previous model potential calculations ${ }^{23-25}$ ( $\mathrm{cf}$. plots of potentials in Krylov et al. ${ }^{1}$ ) although the well depths and the position of the minima are not identical, as will be discussed below. Enlarged plots of the $0^{-}$(II), 1(III), and $0^{+}$(III) potentials in the region around the energy minima are given in Fig. 4, while in Fig. 5 the calculated dipole transition moments for the allowed transitions from the excited states to the ground state in $\mathrm{XeKr}$ are given.

In Table III, some data relevant to the minima of the excited state potentials of $\mathrm{XeKr}$ are given along with the calculated radiative lifetimes for the $v=0,1,2$, and 10 levels of the excited states. The calculated potentials have minima ranging between 598 and $860 \mathrm{~cm}^{-1}$, with $r_{e}$ ranging between 6.9 and 7.8 bohr. Experimental well depths are generally 
TABLE II. Calculated total energies ${ }^{\mathrm{a}}\left(\mathrm{cm}^{-1}\right)$ of excited states of $\mathrm{XeKr}$ with respect to the minimum energy of the ground state.

\begin{tabular}{|c|c|c|c|c|c|c|c|c|}
\hline$R$ (bohr) & $0^{+}(\mathrm{II})$ & $0^{+}(\mathrm{III})$ & 2(I) & $0^{-}(\mathrm{I})$ & $0^{-}$(II) & 1(I) & 1(II) & 1(III) \\
\hline 4.0 & 105486 & 141282 & 135192 & 104610 & 141276 & 104616 & 135809 & 141912 \\
\hline 4.25 & 89715 & 119606 & 113389 & 88971 & 119600 & 88982 & 114051 & 120283 \\
\hline 4.5 & 79818 & 104555 & 98187 & 79144 & 104548 & 79162 & 98874 & 105246 \\
\hline 4.75 & 73793 & 94195 & 87638 & 73144 & 94187 & 73172 & 88358 & 94908 \\
\hline 5.0 & 70280 & 87152 & 80365 & 69627 & 87139 & 69670 & 81120 & 87883 \\
\hline 5.25 & 68335 & 82451 & 75382 & 67665 & 82428 & 67728 & 76170 & 83182 \\
\hline 5.5 & 67331 & 79402 & 71992 & 66631 & 79360 & 66718 & 72815 & 80108 \\
\hline 5.75 & 66841 & 77514 & 69710 & 66111 & 77435 & 66223 & 70570 & 78161 \\
\hline 6.0 & 66607 & 76417 & 68191 & 65857 & 76284 & 65986 & 69094 & 76978 \\
\hline 6.25 & 66503 & 75828 & 67192 & 65731 & 75629 & 65862 & 68146 & 76289 \\
\hline 6.5 & 66453 & 75545 & 66540 & 65667 & 75275 & 65782 & 67557 & 75907 \\
\hline 6.75 & 66441 & 75431 & 66124 & 65634 & 75094 & 65718 & 67203 & 75703 \\
\hline 7.0 & 66440 & 75397 & 65860 & 65614 & 75003 & 65665 & 66997 & 75596 \\
\hline 7.25 & 66459 & 75409 & 65701 & 65604 & 74961 & 65626 & 66881 & 75544 \\
\hline 7.5 & 66481 & 75439 & 65610 & 65604 & 74949 & 65605 & 66822 & 75526 \\
\hline 7.75 & 66523 & 75485 & 65575 & 65616 & 74959 & 65606 & 66802 & 75531 \\
\hline 8.0 & 66570 & 75541 & 65569 & 65638 & 74987 & 65620 & 66808 & 75555 \\
\hline 8.25 & 66620 & 75596 & 65589 & 65670 & 75021 & 65648 & 66831 & 75588 \\
\hline 8.5 & 66678 & 75657 & 65625 & 65706 & 75064 & 65685 & 66866 & 75629 \\
\hline 8.75 & 66732 & 75714 & 65667 & 65743 & 75105 & 65723 & 66903 & 75669 \\
\hline 9.0 & 66782 & 75763 & 65714 & 65778 & 75144 & 65761 & 66941 & 75709 \\
\hline 9.5 & 66865 & 75850 & 65792 & 65837 & 75216 & 65825 & 67007 & 75777 \\
\hline 10.0 & 66916 & 75903 & 65846 & 65876 & 75261 & 65868 & 67050 & 75821 \\
\hline 10.5 & 66958 & 75946 & 65891 & 65909 & 75300 & 65904 & 67086 & 75857 \\
\hline 11.0 & 66995 & 75985 & 65932 & 65942 & 75337 & 65939 & 67121 & 75892 \\
\hline 11.5 & 67032 & 76022 & 65972 & 65976 & 75373 & 65975 & 67156 & 75928 \\
\hline 12.0 & 67064 & 76055 & 66007 & 66008 & 75406 & 66008 & 67189 & 75960 \\
\hline 12.5 & 67092 & 76084 & 66037 & 66036 & 75435 & 66036 & 67217 & 75989 \\
\hline 13.0 & 67115 & 76107 & 66060 & 66057 & 75458 & 66058 & 67239 & 76011 \\
\hline 14.0 & 67155 & 76146 & 66100 & 66095 & 75497 & 66096 & 67278 & 76050 \\
\hline 15.0 & 67186 & 76177 & 66132 & 66126 & 75530 & 66127 & 67309 & 76082 \\
\hline 16.0 & 67209 & 76201 & 66155 & 66148 & 75554 & 66150 & 67333 & 76106 \\
\hline 17.0 & 67223 & 76217 & 66169 & 66163 & 75570 & 66165 & 67347 & 76120 \\
\hline 18.0 & 67232 & 76225 & 66179 & 66174 & 75577 & 66175 & 67357 & 76129 \\
\hline 19.0 & 67240 & 76233 & 66186 & 66182 & 75584 & 66183 & 67364 & 76136 \\
\hline 20.0 & 67245 & 76238 & 66191 & 66187 & 75589 & 66188 & 67369 & 76141 \\
\hline 100.0 & 67264 & 76257 & 66206 & 66203 & 75612 & 66203 & 67387 & 76161 \\
\hline
\end{tabular}

${ }^{\mathrm{a}}$ Unshifted energies.

smaller than the theoretical values, with a variation in the proposed $r_{e}$ as well, as mentioned above in the Introduction. For the $1(\mathrm{I})$ state [also denoted $\left.1\left({ }^{3} P_{2}\right)\right]$ the well-depth value of $138 \pm 50 \mathrm{~cm}^{-1}$ at an $r_{0}$ value of $6.05-6.24 \mathrm{bohr}$ has been

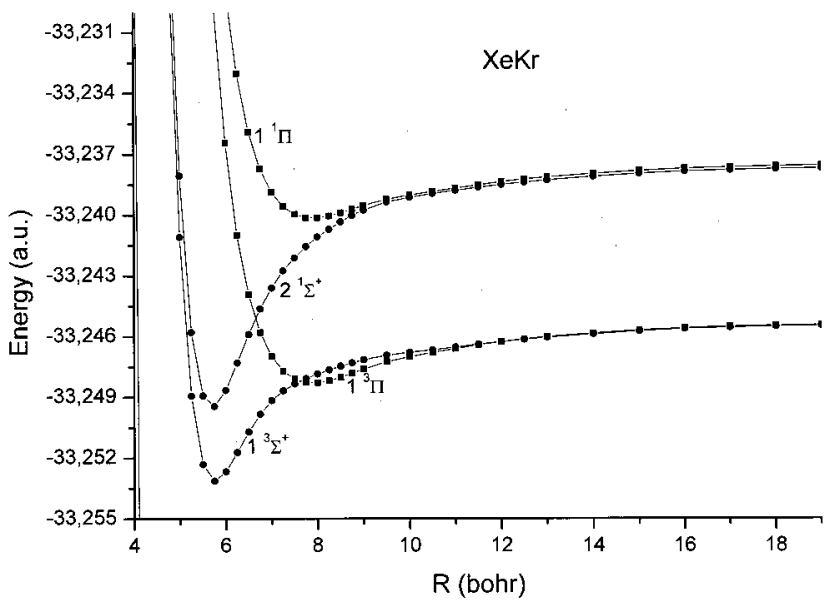

FIG. 1. Potential energy curves for the $\Lambda$-S states of XeKr. proposed $^{16}$ for $\mathrm{XeKr}$ in the solid phase, corresponding to emission at $7.95 \mathrm{eV}$, while other observations of the $1(\mathrm{I})$ $\rightarrow X 0^{+}$(I) transition also place it at $153-156 \mathrm{~nm}^{22}$ (i.e., a transition energy of 8.1-7.95 eV). The present calculations obtain a higher value for the vertical transition energy of this state $(8.3 \mathrm{eV})$ at the calculated minimum $(R=7.6 \mathrm{bohr})$. The dipole transition moment for the $1(\mathrm{I}) \rightarrow X 0^{+}$(I) (cf. Fig. 5) shows a maximum at $6.0 \mathrm{bohr}$ and lower values in the region of the minimum. As a result the computed radiative lifetime for this state is $21.0 \mu \mathrm{s}$ for $v=0$, decreasing to $0.6 \mu \mathrm{s}$ for $v=10$ (cf. Table III). Thus the present calculations are not in agreement with the results of previous model calculations of the radiative lifetimes ${ }^{25}$ in which the experimental potential was used for the region near the minimum and according to which the lifetime of the 1(I) state is under $100 \mathrm{~ns}$ and increases with vibrational quantum number. However, a plot of the transition probability obtained using the $1(I) \rightarrow X 0^{+}(I)$ dipole transition moments of the present work (Fig. 5), as given in Fig. 6, is very similar to the plot of the radiation width in the previous model calculations ${ }^{25}$ showing a steep maximum at $6.0 \mathrm{bohr}$, at which internuclear distance the transition energy is $8.04 \mathrm{eV}$, well within the range of ob- 


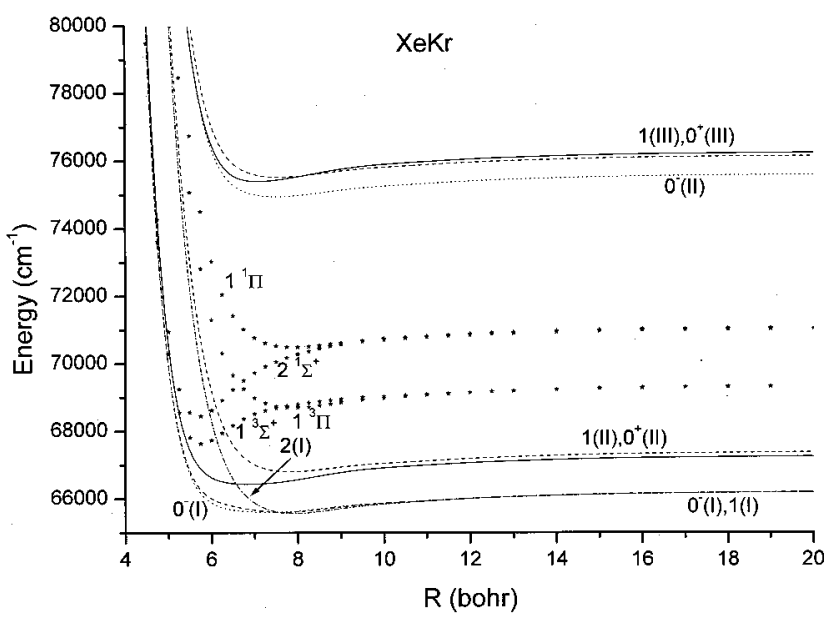

FIG. 2. Potential energy curves for $\Omega$ states of $\mathrm{Xe}$ * $\left(5 p^{5} 6 s^{1,3} P\right)$ $+\mathrm{Kr}\left(2 p^{6}{ }^{1} S\right)$.

served values. In view of the difficulties in determining a precise value for the $r_{e}$ of this state and with only a single experimental deduction, it is difficult to draw further conclusions.

Most of the available spectroscopic work involves the excited states observed near the first resonance line of $\mathrm{Xe}^{*}$, i.e., $0^{+}$(II) and 1(II). Generally shallow wells are proposed: Depending on the potential parameters employed ${ }^{2}$ well depths of $120 \mathrm{~cm}^{-1}$ at 9.03 bohr or $160 \mathrm{~cm}^{-1}$ at $8.2 \mathrm{bohr}$ are estimated on the basis of the absorption spectra. ${ }^{2}$ Another estimate of the minimal dissociation energy for these states is $153 \mathrm{~cm}^{-1}$ at a transition energy of $67892 \mathrm{~cm}^{-1}$. For the 1(II) state Pibel et al. ${ }^{19}$ propose a well depth of $52.2 \mathrm{~cm}^{-1}$ at $5.24 \AA$ (9.90 bohr), while for the $0^{+}$(II) state they propose a double well potential with an inner minimum of $624 \mathrm{~cm}^{-1}$ at $3.09 \AA$ (5.8 bohr) and an outer minimum of $101 \mathrm{~cm}^{-1}$ at 9.6 bohr. ${ }^{19}$ Model Hamiltonian calculations obtained $440 \mathrm{~cm}^{-1}$ at $r_{e}=8.20 \mathrm{bohr}$ for 1(II) and a single minimum of $920 \mathrm{~cm}^{-1}$ at $r_{e}=6.71 \mathrm{bohr}$ for $0^{+}$(II). ${ }^{23,24}$ Similarily, in the present work, there is no evidence for a double well potential for $0^{+}(\mathrm{II})$, with a single minimum of $585 \mathrm{~cm}^{-1}$ found for this

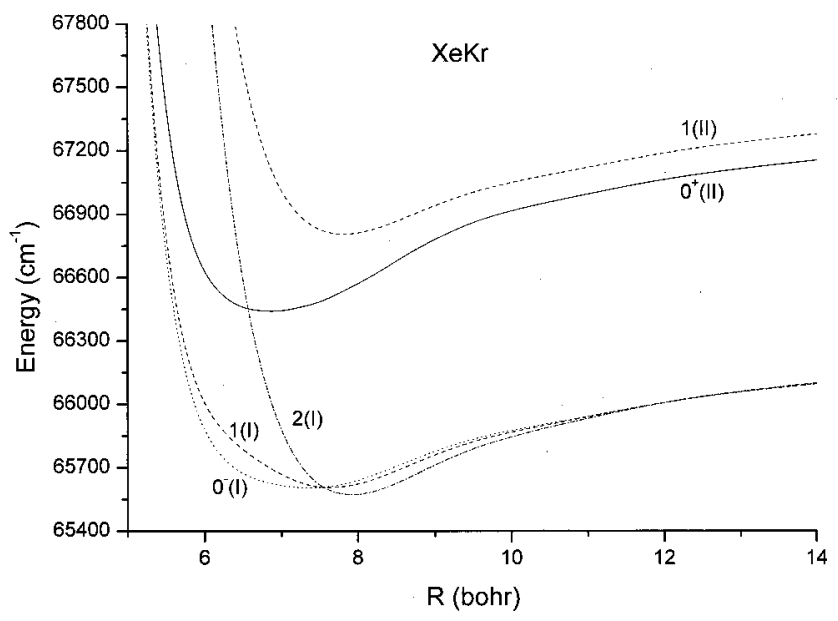

FIG. 3. Potential energy curves of the $0^{-}$(I), 1(I), 2(I), 1(II), and $0^{+}$(II) states of $\mathrm{Xe}^{*} \mathrm{Kr}$.

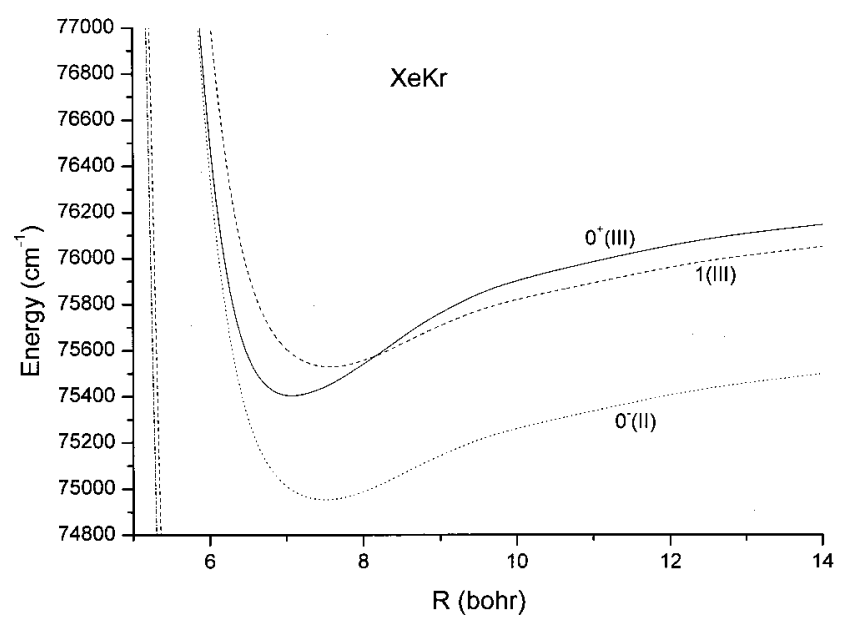

FIG. 4. Potential energy curves of the $0^{-}$(II), 1 (III), and $0^{+}$(III) states of $\mathrm{Xe}{ }^{*} \mathrm{Kr}$.

state at 6.9 bohr. It is possible that the experimental deductions ${ }^{19}$ might change if the potentials and transition moments of the present work are employed in the analysis of the spectra. Finally, recent experimental work involving emission near $147 \mathrm{~nm}$ report that it is not possible to distinguish between emission from 1 (II) and from the $0^{+}$(II) states. ${ }^{1,22}$ These authors favor a well depth of $170 \mathrm{~cm}^{-1}$ at 8.3 bohr for 1 (II). The calculated $r_{e}, \Delta E_{V}$ and $D_{e}$ of the present work for these states (cf. Table III) are in reasonably good agreement with experiment.

For the higher states of $\mathrm{XeKr}$ computed in the present work, $0^{-}$(II), 1(III), and $0^{+}$(III) there are no previous theoretical calculations. A bound excited state near the second resonance line of $\mathrm{Xe}^{*}$ with well depth of $333 \mathrm{~cm}^{-1}$ at a transition energy of $77185.1 \mathrm{~cm}^{-1}$ has been proposed in an early absorption spectroscopy study. ${ }^{3}$ More recent experimental estimates ${ }^{18}$ of the interatomic potentials of the 1(III) and $0^{+}$(III) states yield a dissociation energy of $1445 \mathrm{~cm}^{-1}$ for the 1 (III) state at $5.7 \mathrm{bohr}$ and $54 \mathrm{~cm}^{-1}$ for the $0^{+}$(III) state at 6.9 bohr, for transition energies 75899.5 and $77293.0 \mathrm{~cm}^{-1}$, respectively. Subsequent work ${ }^{20}$ reversed the

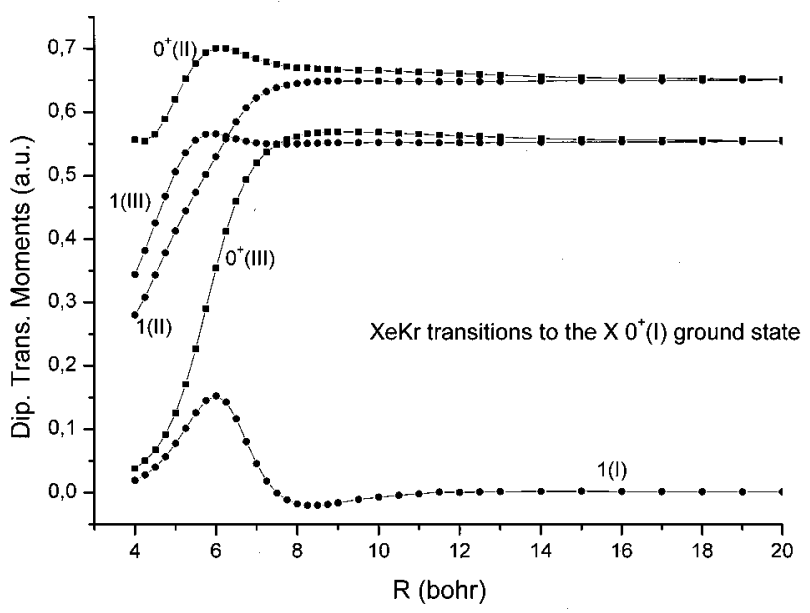

FIG. 5. Calculated transition moments between the excited and the ground state of XeKr. 
TABLE III. Calculated molecular constants of excited states of XeKr.

\begin{tabular}{|c|c|c|c|c|c|c|c|}
\hline State & $\begin{array}{c}r_{e} \\
\text { bohr }\end{array}$ & $\begin{array}{c}B_{e} \\
\mathrm{~cm}^{-1}\end{array}$ & $\begin{array}{c}\omega_{e} \\
\mathrm{~cm}^{-1}\end{array}$ & $\begin{array}{l}\Delta E_{v}{ }^{a} \\
\mathrm{~cm}^{-1}\end{array}$ & $\begin{array}{c}D_{e} \\
\mathrm{~cm}^{-1}\end{array}$ & $\begin{array}{c}E v=0^{\mathrm{b}} \\
\mathrm{cm}^{-1}\end{array}$ & $\begin{array}{c}\tau_{\mathrm{rad}} \\
(v=0,1,2,10)\end{array}$ \\
\hline $0^{-}(\mathrm{I})$ & 7.38 & 0.022 & 40.27 & 67288 & 599 & 67344 & $\cdots$ \\
\hline $1(\mathrm{I})$ & 7.60 & 0.021 & 40.63 & 67326 & 598 & 67347 & $\begin{array}{r}21.0 \mu \mathrm{s}, 6.2 \mu \mathrm{s}, \\
2.9 \mu \mathrm{s}, 0.6 \mu \mathrm{s}\end{array}$ \\
\hline $2(\mathrm{I})$ & 7.94 & 0.019 & 35.21 & 67300 & 637 & 67315 & $\ldots$ \\
\hline $0^{+}(\mathrm{II})$ & 6.91 & 0.025 & 45.73 & 67822 & 824 & 68182 & $\begin{array}{l}3.4 \mathrm{~ns}, 3.7 \mathrm{~ns} \\
4.3 \mathrm{~ns}, 3.5 \mathrm{~ns}\end{array}$ \\
\hline 1(II) & 7.80 & 0.019 & 31.81 & 68528 & 585 & 68547 & $\begin{array}{r}12.5 \mathrm{~ns}, 4.9 \mathrm{~ns}, \\
3.9 \mathrm{~ns}, 3.7 \mathrm{~ns}\end{array}$ \\
\hline $0^{-}(\mathrm{II})$ & 7.51 & 0.021 & 15.27 & 76678 & 663 & 76694 & $\ldots$ \\
\hline $0^{+}(\mathrm{III})$ & 7.03 & 0.024 & 22.98 & 76888 & 860 & 77147 & $\begin{array}{l}4.2 \mathrm{~ns}, 5.0 \mathrm{~ns} \\
5.7 \mathrm{~ns}, 3.7 \mathrm{~ns}\end{array}$ \\
\hline 1(III) & 7.67 & 0.021 & 33.14 & 77253 & 635 & 77271 & $\begin{array}{r}10.4 \mathrm{~ns}, 4.8 \mathrm{~ns}, \\
3.8 \mathrm{~ns}, 3.5 \mathrm{~ns}\end{array}$ \\
\hline
\end{tabular}

${ }^{a}$ With respect to the ground-state energy at $r_{e}$ of the excited state, including the shift of $1731 \mathrm{~cm}^{-1}$.

${ }^{b}$ With respect to the ground-state minimum energy, including the shift of $1731 \mathrm{~cm}^{-1}$.

above assignment, proposing that the lower energy transition (to the state with the deeper minimum) is the $0^{+}(\mathrm{III})-X$ $0^{+}(I)$. The transition energies of the present work, 76888 and $77253 \mathrm{~cm}^{-1}$ for the $0^{+}$(III) and 1(III), respectively (cf. Table III), do show that the potential energy curve of the $0^{+}$(III) state near the minimum lies below that of the 1(III) state (cf. also Fig. 4), and are in good agreement with the observed transition energies. Furthermore, the deepest minima calculated are those of the $0^{+}$(III) and $0^{+}$(II) states (cf. Table III). There is difficulty in comparing the calculated $r_{e}$ values for 1 (III) and $0^{+}$(III) with the experimental. ${ }^{18,20}$ It might be noted that the location of the proposed deep minimum at relatively short bond length for the $0^{+}$(III) state, ${ }^{20}$ and also similarily for the $0^{+}$(II) state, ${ }^{19}$ is close to the position of the minimum in the potential of the $2^{1} \Sigma^{+}$state (cf. Fig. 1), which along with $1^{3} \Pi$ (mostly) and $X^{1} \Sigma^{+}$interact via spin-orbit coupling to produce the $0^{+}$states. Similarily, the available experimental $r_{e}$ estimate for the 1 (I) state ${ }^{16}$ is closer to that of the minimum in the $1^{3} \Sigma^{+}$state (cf. Fig. 1) than to the value calculated for 1(I) (cf. Table III).

As might be expected, large dipole transition moments (cf. Fig. 5) and correspondingly short lifetimes (a few nano-

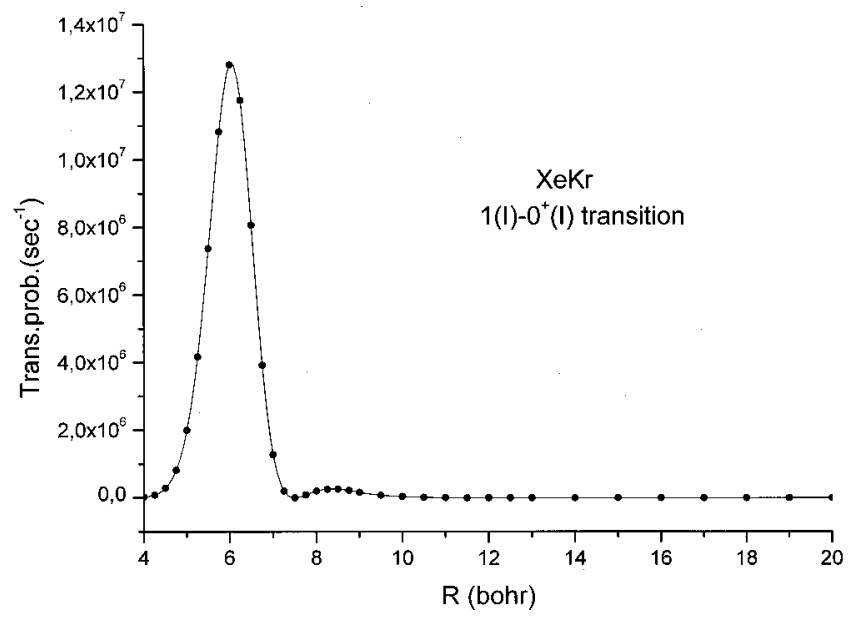

FIG. 6. Calculated radiative transition probability for the $1(\mathrm{I}) \rightarrow X 0^{+}$(I) transition. seconds) are obtained for the $0^{+}$(II), $1(\mathrm{II}), 0^{+}$(III), and 1(III) states (cf. Table III) that correlate with atomic limits which have dipole-allowed transitions to the ground state.

\section{B. Potential energy curves of ArNe}

As mentioned in the Introduction, a previous study has been devoted to the potential energy curves of the $\Omega$ states of $\operatorname{Ar}\left(3 p^{6}{ }^{1} S, 3 p^{5} 4 s^{1,3} P\right)+\mathrm{Ne}\left(2 p^{6}{ }^{1} S\right)$ in which only a single $s$ and a single set of $p$ diffuse functions optimized at the dissociation limits with respect to the atomic excitation energies were employed. ${ }^{14}$ Those calculations obtained both good excitation energies, without the necessity of any shift, and transition moments at the dissociation limits, a reasonably accurate ground-state potential and well depths of about 800 $\mathrm{cm}^{-1}$ at internuclear distances of 7.5-8.5 bohr for the excited state potentials. However, a subsequent theoretical study of ArHe and $\mathrm{HeNe}^{15}$ has shown that the results obtained with the treatment adopted in the work on $\mathrm{ArNe}^{14}$ were not stable with respect to changes in the Rydberg basis set and that it is necessary to have a triple-zeta diffuse basis in order to obtain converged results.

The results of the present calculations on the $\Omega$ states of ArNe are listed in Table IV, while the potential energy curves are shown in Fig. 7 and the transition moments to the ground state in Fig. 8. As shown in Fig. 7, the potential energy curves for both the $\Lambda$-S and the $\Omega$ excited states are calculated to be totally repulsive. While the existence of very shallow minima $\left(\sim 100 \mathrm{~cm}^{-1}\right)$ cannot be excluded, the present calculations do not support the existence of any minima of $800 \mathrm{~cm}^{-1}$ depth indicated in the previous study. This result underscores the importance of employing a flexible basis for the Rydberg orbitals in such calculations. More generally, the lack of any experimental information on the excited states of ArNe makes it quite difficult to describe these systems on a definitive basis.

\section{CONCLUSIONS}

Relativistic core-potential calculations have been carried out on $\Omega$ states resulting from the interaction of $\mathrm{Xe}^{*}$ 
TABLE IV. Calculated total energies ${ }^{\mathrm{a}}\left(\mathrm{cm}^{-1}\right)$ of excited states of ArNe with respect to the minimum energy of the ground state.

\begin{tabular}{crrrrrrrr}
\hline \hline$R$ (bohr) & $0^{+}(\mathrm{II})$ & $0^{+}(\mathrm{III})$ & \multicolumn{1}{c}{$2(\mathrm{I})$} & $0^{-}(\mathrm{I})$ & $0^{-}(\mathrm{II})$ & $1(\mathrm{I})$ & $1(\mathrm{II})$ & $1(\mathrm{III})$ \\
\hline 3.50 & 101000 & 112930 & 111928 & 100136 & 112928 & 100138 & 112277 & 113882 \\
3.75 & 97058 & 104731 & 103709 & 96108 & 104727 & 96113 & 104070 & 105733 \\
4.00 & 95145 & 99817 & 98762 & 94115 & 99803 & 94125 & 99134 & 100838 \\
4.25 & 94161 & 96863 & 95733 & 93089 & 96816 & 93109 & 96121 & 97854 \\
4.75 & 92888 & 94230 & 92676 & 91980 & 93908 & 92025 & 93137 & 94877 \\
5.00 & 92563 & 93921 & 92162 & 91737 & 93448 & 91782 & 92662 & 94387 \\
5.25 & 92292 & 93717 & 91801 & 91539 & 93133 & 91577 & 92342 & 94052 \\
5.50 & 92079 & 93569 & 91540 & 91375 & 92905 & 91404 & 92115 & 93812 \\
5.75 & 91902 & 93448 & 91331 & 91231 & 92723 & 91251 & 91936 & 93622 \\
6.00 & 91745 & 93338 & 91154 & 91097 & 92564 & 91110 & 91782 & 93456 \\
6.25 & 91606 & 93241 & 90999 & 90977 & 92427 & 90982 & 91648 & 93311 \\
6.50 & 91472 & 93143 & 90852 & 90858 & 92294 & 90856 & 91519 & 93171 \\
6.75 & 91353 & 93052 & 90725 & 90749 & 92177 & 90743 & 91404 & 93047 \\
7.00 & 91257 & 92977 & 90623 & 90659 & 92081 & 90649 & 91311 & 92947 \\
7.25 & 91181 & 92911 & 90544 & 90581 & 92003 & 90571 & 91233 & 92869 \\
7.50 & 91125 & 92861 & 90486 & 90519 & 91943 & 90510 & 91172 & 92810 \\
7.75 & 91097 & 92828 & 90459 & 90487 & 91914 & 90480 & 91141 & 92779 \\
8.00 & 91080 & 92806 & 90444 & 90465 & 91895 & 90460 & 91121 & 92760 \\
8.25 & 91065 & 92787 & 90431 & 90447 & 91879 & 90443 & 91104 & 92743 \\
8.50 & 91056 & 92773 & 90423 & 90436 & 91869 & 90432 & 91093 & 92732 \\
8.75 & 91048 & 92763 & 90415 & 90427 & 91861 & 90424 & 91084 & 92723 \\
9.00 & 91041 & 92753 & 90409 & 90420 & 91854 & 90417 & 91077 & 92715 \\
9.50 & 91023 & 92733 & 90391 & 90405 & 91839 & 90401 & 91062 & 92699 \\
10.0 & 91001 & 92712 & 90370 & 90389 & 91820 & 90384 & 91044 & 92681 \\
11.0 & 90982 & 92689 & 90352 & 90356 & 91795 & 90355 & 91017 & 92661 \\
12.0 & 91000 & 92702 & 90372 & 90367 & 91810 & 90369 & 91030 & 92673 \\
13.0 & 91005 & 92710 & 90375 & 90373 & 91815 & 90375 & 91036 & 92677 \\
14.0 & 91010 & 92714 & 90381 & 90380 & 91821 & 90380 & 91042 & 92683 \\
15.0 & 91014 & 92715 & 90386 & 90384 & 91825 & 90384 & 91046 & 92687 \\
16.0 & 91017 & 92716 & 90389 & 90384 & 91827 & 90385 & 91047 & 92691 \\
18.0 & 91017 & 92715 & 90390 & 90383 & 91826 & 90385 & 91047 & 92693 \\
20.0 & 91024 & 92721 & 90397 & 90389 & 91833 & 90391 & 91054 & 92701 \\
\hline \hline
\end{tabular}

${ }^{\mathrm{a}}$ Unshifted energies.

$\left(5 p^{5} 6 s,{ }^{3} P,{ }^{1} P\right)$ with ground state $\mathrm{Kr}$ atoms as well as for the system $\mathrm{Ar}^{*}\left(3 p^{5} 4 s,{ }^{3} P,{ }^{1} P\right)$ with ground state $\mathrm{Ne}$, using different basis sets and configuration interaction procedures. The present calculations on $\mathrm{XeKr}$ obtain shallow minima for the excited states of this system, in generally good qualitative agreement with previous model Hamiltonian calculations. However, the results differ in details such as the depth of the minima, the $r_{e}$ values and the radiative lifetime of the

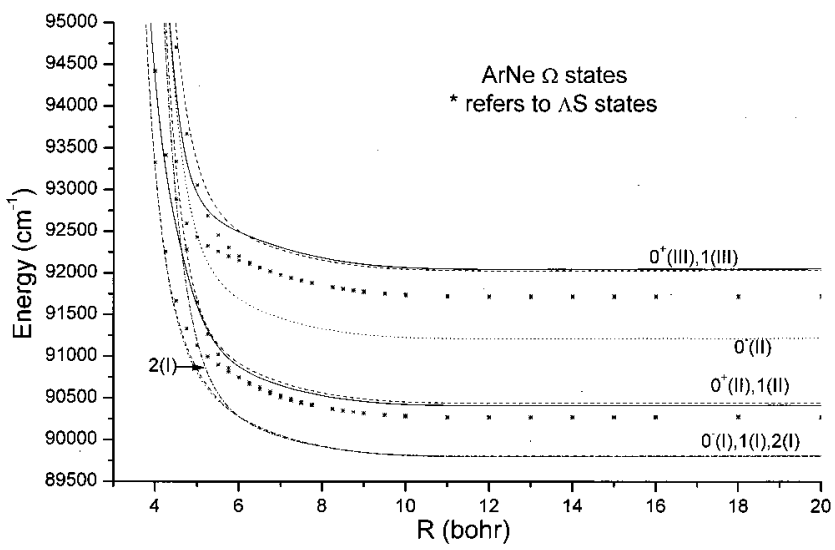

FIG. 7. Potential energy curves for $\Omega$ states of $\mathrm{Ar}^{*}\left(3 p^{5} 4 s^{1,3} \mathrm{P}\right)$ $+\mathrm{Ne}\left(2 p^{6}{ }^{1} S\right)$.
1(I) state. It is rather difficult to make quantitative interpretations for the available experimental data on the $\Omega$ states of $\mathrm{XeKr}$ calculated in the present work because there are considerable variations with regard to the location of the minima among different experimental studies.

The present calculations on ArNe obtain totally repulsive potential energy curves, showing that our previously reported potentials for this system are erroneous. This result indicates that great care is required in calculations of these systems,

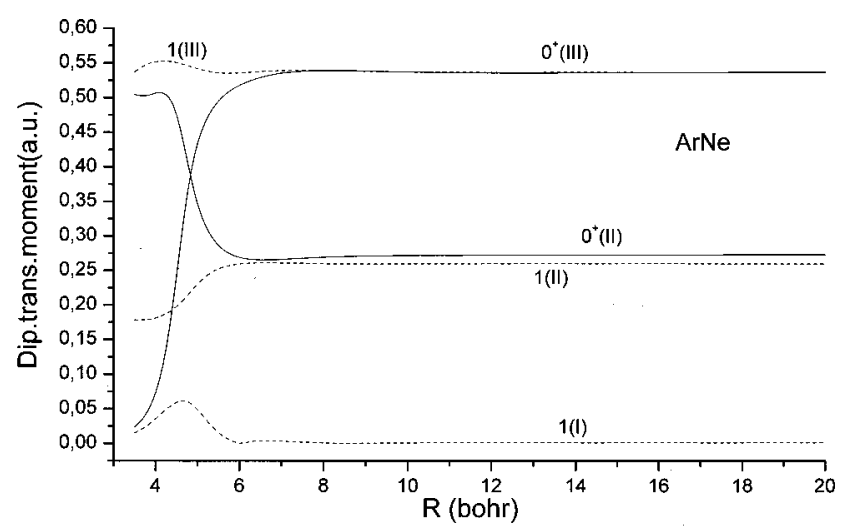

FIG. 8. Calculated transition moments between the excited and the ground state of ArNe. 
particularily in the choice of the diffuse basis functions employed to describe their Rydberg states.

\section{ACKNOWLEDGMENTS}

The present work was supported by NATO CLG 977379 and INTAS OPEN99-0039 as well as from the Verein der Chemischen Industrie.

${ }^{1}$ B. Krylov, G. Gerasimov, A. Arnesen, R. Hallin, and F. Heijkenskjold, Eur. Phys. J. D 8, 227 (2000).

${ }^{2}$ M. C. Castex, J. Chem. Phys. 66, 3854 (1977).

${ }^{3}$ D. E. Freeman, K. Yoshino, and Y. Tanaka, J. Chem. Phys. 67, 3462 (1977).

${ }^{4}$ A. Gedanken, J. Jortner, B. Raz, and A. Szöke, J. Chem. Phys. 57, 3456 (1972).

${ }^{5}$ O. Cheshnovsky, B. Raz, and J. Jortner, J. Chem. Phys. 59, 3301 (1973).

${ }^{6}$ Y. Salamero, A. Birot, H. Brunet, H. Dijols, J. Galy, P. Millet, and J. P. Montagne, J. Chem. Phys. 74, 288 (1981).

${ }^{7}$ T. Efthimiopoulos, D. Zouridis, and D. Ulrich, J. Phys. D 30, 1754 (1997).

${ }^{8}$ V. Kemper, G. Rieke, F. Veith, and L. Zehnle, J. Phys. B 9, 3081 (1976).

${ }^{9}$ D. D. Khadka, Y. Fukuchi, M. Kitajima, K. Hidaka, N. Kouchi, and Y. Hatano, J. Chem. Phys. 107, 2386 (1997).

${ }^{10}$ F. Spiegelman, F. X. Gadea, and M. C. Castex, Chem. Phys. 145, 173 (1990).

${ }^{11}$ A. P. Hickman, D. L. Huestis, and R. P. Saxon, J. Chem. Phys. 96, 2099 (1991).

${ }^{12}$ J. F. Ogilvie and Y. H. Wang, J. Mol. Struct. 291, 313 (1993).

${ }^{13}$ S. M. Cybulski and R. R. Toczylowski, J. Chem. Phys. 111, 10520 (1999).

${ }^{14}$ I. D. Petsalakis, R. J. Buenker, H.-P. Lieberman, A. Alekseyev, A. Z. Devdariani, and G. Theodorakopoulos, J. Chem. Phys. 113, 5812 (2000).

${ }^{15}$ I. D. Petsalakis, G. Theodorakopoulos, H.-P. Lieberman, and R. J. Buenker, J. Chem. Phys. 115, 6365 (2001).

${ }^{16}$ G. Nowak and J. Fricke, J. Phys. B 18, 1355 (1985).

${ }^{17}$ P. Laporte, P. Gurtler, E. Morikawa, R. Reininger, and V. Saile, Europhys. Lett. 9, 533 (1989).
${ }^{18}$ T. Tsuchizawa, K. Yamanouchi, and S. Tsuchiya, J. Chem. Phys. 92, 1560 (1990).

${ }^{19}$ C. D. Pibel, K. Yamanouchi, J. Miyawaki, and S. Tsuchiya, J. Chem. Phys. 101, 10242 (1994).

${ }^{20}$ D. M. Mao, X. K. Hu, S. S. Dimov, and R. H. Lipson, J. Phys. B 29, L89 (1996).

${ }^{21}$ G. Gerasimov, R. Khallin, and B. Krylov, Opt. Spectrosc. 88, 176 (2000).

${ }^{22}$ A. Morozov, B. Krylov, G. Gerasimov, R. Hallin, and A. Arnesen, Eur. Phys. J. D 11, 379 (2000).

${ }^{23}$ A. Z. Devdariani, A. L. Zagrebin, and K. B. Blagoev, Ann. Phys. (Paris) 14, 467 (1989).

${ }^{24}$ A. L. Zagrebin and N. A. Pavlovskaya, Opt. Spectrosc. 69, 320 (1990).

${ }^{25}$ A. L. Zagrebin and S. I. Tserkovnyi, Chem. Phys. Lett. 239, 136 (1995).

${ }^{26}$ A. B. Alekseyev, H.-P. Lieberman, R. J. Buenker, G. Hirsch, and Y. Li, J. Chem. Phys. 100, 8956 (1994).

${ }^{27}$ A. B. Alekseyev, R. J. Buenker, H.-P. Lieberman, and G. Hirsch, J. Chem. Phys. 100, 2989 (1994).

${ }^{28}$ R. J. Buenker, A. B. Alekseyev, H.-P. Lieberman, R. Lingott, and G. Hirsch, J. Chem. Phys. 108, 3400 (1998).

${ }^{29}$ R. J. Buenker and R. A. Phillips, J. Mol. Struct.: THEOCHEM 123, 291 (1985).

${ }^{30}$ S. Krebs and R. J. Buenker, J. Chem. Phys. 103, 5613 (1995).

${ }^{31}$ L. A. LaJohn, P. A. Christiansen, R. B. Ross, T. Atashroo, and W. C. Ermler, J. Chem. Phys. 87, 2812 (1987).

${ }^{32}$ M. M. Hurley, L. F. Pacios, P. A. Christiansen, R. B. Ross, and W. C. Ermler, J. Chem. Phys. 84, 6840 (1986).

${ }^{33}$ S. R. Langhoff and E. R. Davidson, Int. J. Quantum Chem. 8, 61 (1974).

${ }^{34}$ L. F. Pacios and P. A. Christiansen, J. Chem. Phys. 82, 2664 (1985).

${ }^{35}$ A. D. McLean and G. S. Chandler, J. Chem. Phys. 72, 5639 (1980).

${ }^{36}$ T. H. Dunning, J. Chem. Phys. 55, 716 (1971).

${ }^{37}$ C. E. Moore, Atomic Energy Levels As Derived From the Analyses of Optical Spectra, Circular of the National Bureau of Standards 467, June 1949.

${ }^{38}$ D. Hausamann and H. Morgner, Mol. Phys. 54, 1085 (1985). 